\title{
Adaptive Neural Tracking Control for Discrete-Time Switched Nonlinear Systems with Dead Zone Inputs
}

\author{
Jidong Wang, Lengxue Zhu, and Xiaoping Si \\ School of Electrical Engineering, North China University of Water Resources and Electric Power, Zhengzhou 450011, China \\ Correspondence should be addressed to Jidong Wang; kfwjd@ncwu.edu.cn
}

Received 13 December 2016; Revised 26 February 2017; Accepted 1 March 2017; Published 23 March 2017

Academic Editor: Sigurdur F. Hafstein

Copyright (C) 2017 Jidong Wang et al. This is an open access article distributed under the Creative Commons Attribution License, which permits unrestricted use, distribution, and reproduction in any medium, provided the original work is properly cited.

\begin{abstract}
In this paper, the adaptive neural controllers of subsystems are proposed for a class of discrete-time switched nonlinear systems with dead zone inputs under arbitrary switching signals. Due to the complicated framework of the discrete-time switched nonlinear systems and the existence of the dead zone, it brings about difficulties for controlling such a class of systems. In addition, the radial basis function neural networks are employed to approximate the unknown terms of each subsystem. Switched update laws are designed while the parameter estimation is invariable until its corresponding subsystem is active. Then, the closed-loop system is stable and all the signals are bounded. Finally, to illustrate the effectiveness of the proposed method, an example is employed.
\end{abstract}

\section{Introduction}

In the past few decades, intelligent control of uncertain nonlinear systems has attracted much more attention. Based on the universal approximation properties of neural networks (NNs) and fuzzy logic systems (FLSs), they are always used to approximate the unknown system functions or the control inputs. So far, there are a lot of results in intelligent control [1-6]. For example, the NN-based adaptive control methods were proposed for uncertain nonlinear systems in $[7,8]$ by using backstepping technique. The above results are all about nonlinear systems in continuous-time form. On the contrary, many researchers devoted much effort to study the adaptive control problem of discrete-time systems by using intelligent methods on the basis of these works [9-11].

However, the previous results do not consider the switching phenomenon of the system. Actually, the switching phenomenon often exists in practical systems. We call this class of systems switched systems. Switched systems, which are used to model a wide variety of physical systems, consist of a family of continuous-time or discrete-time subsystems and a switching rule to govern the switching between the subsystems. Generally, the stability and stabilisation problems are the main concerns in the study of switched systems [12-16]. Subsequently, adaptive control and intelligent control of switched systems have been studied more and more [17$22]$. But to the best of our knowledge, there are no results on discrete-time switched systems, and let alone results on discrete-time switched nonlinear systems.

In addition, the dead zone input, as one of the most important input nonlinearity, widely exists in lots of practical systems. The existence of the dead zone input may damage the system performance or even destroy the system stability. Thus, the related robust control attracted high attention $[8$, 23-25]. Since the dead zone parameters are poorly known in most practical systems, adaptive control techniques are naturally used to deal with this problem. An adaptive tracking control strategy was proposed for a nonlinear system with nonsymmetric dead zone input having unknown but bounded parameters in [26].

Motivated by the above discussion, we study the adaptive neural control problem of a class of discrete-time switched nonlinear systems with dead zone inputs under arbitrary switching signals. We use the radial basis function neural networks to approximate the unknown terms of each subsystem. Dead zone inputs of subsystems and switched update laws are designed such that the closed-loop system is stable and all the signals are bounded. Finally, an example is employed to illustrate the effectiveness of the proposed method. 
The main contributions of this paper compared with the existing results on switched and nonswitched nonlinear systems are in two aspects:

(1) Compared with the existing results [8, 23-25], in which the considered systems are all nonswitched systems or continuous-time switched systems, in this paper, we study a discrete-time switched uncertain nonlinear system.

(2) The dead zone input is considered in the discrete-time switched uncertain nonlinear system. As far as we know, there are no results on discrete-time switched systems with dead zone inputs. This is mainly due to the complicated framework of the discrete-time switched nonlinear systems, the existence of the dead zone, and the interaction between the system structure and switching.

\section{System Description}

In this paper, we consider the following discrete-time switched uncertain nonlinear system:

$$
\begin{gathered}
\xi_{1}(k+1)=\xi_{2}(k), \\
\vdots \\
\xi_{n-1}(k+1)=\xi_{n}(k), \\
\xi_{n}(k+1)=f_{\sigma}(\xi(k))+g_{\sigma}(\xi(k)) u_{\sigma}(k), \\
y(k)=\xi_{1}(k),
\end{gathered}
$$

where $\xi(k)=\left[\xi_{1}(k), \xi_{2}(k), \ldots, \xi_{n}(k)\right]^{T} \in \mathfrak{R}^{n}$ and $y \in \Re$ are the system state and output, respectively. Moreover, the state of switched system (1) is assumed not to jump at the switching instants, which is a standard assumption in the switched system [27]. $\sigma$ is the switching signal taking values in $P=\{1,2, \ldots, p\}$ with $p$ being the subsystems number of switched system (1). $f_{i}(x(k))$ and $g_{i}(x(k)), i \in P$ are unknown smooth functions. $u_{i}(k)$ is the dead zone output of the $i$ th subsystem, which is described by

$$
\begin{aligned}
u_{i}(k)= & D\left(v_{i}(k)\right) \\
= & \begin{cases}h_{i r}\left(v_{i}(k)-w_{i r}\right), & \text { if } v_{i}(k) \geq w_{i r}, \\
0, & \text { if }-w_{i l}<v_{i}(k)<w_{i r}, \\
h_{i l}\left(v_{i}(k)+w_{i l}\right), & \text { if } v_{i}(k) \leq-w_{i l} .\end{cases}
\end{aligned}
$$

In (2), $v_{i}(k)$ is the dead zone input of the $i$ th subsystem. $h_{i r}$ and $h_{i l}$ stand for the right and left slopes of the dead zone characteristic, respectively. $w_{i r}$ and $w_{i l}$ represent the breakpoints of the input nonlinearity.

According to [28], the dead zone can be further expressed as

$$
u_{i}(k)=D\left(v_{i}(k)\right)=h_{i}(k) v_{i}(k)+w_{i}(k), \quad i \in P,
$$

where

$$
\begin{aligned}
& h_{i}(k)= \begin{cases}h_{i r}, & \text { if } v_{i}(k)>0, \\
h_{i l}, & \text { if } v_{i}(k) \leq 0,\end{cases} \\
& w_{i}(k)= \begin{cases}-h_{i r} w_{i r}, & \text { if } v_{i}(k) \geq w_{i r}, \\
-h_{i}(k) v_{i}(k), & \text { if }-w_{i l}<v_{i}(k)<w_{i r}, \\
h_{i l} w_{i l}, & \text { if } v_{i}(k) \leq-w_{i l} .\end{cases}
\end{aligned}
$$

Assumption 1. The parameters of dead zone are all positive, and they are unknown but bounded, that is, $\underline{h}_{i}=\min \left\{h_{i r}, h_{i l}\right\}$, $\bar{h}_{i}=\max \left\{h_{i r}, h_{i l}\right\}$, and $\left|w_{i}(k)\right| \leq \bar{w}_{i}$.

From Assumption 1, we can easily know that

$$
\begin{gathered}
\underline{h}_{i} \leq h_{i}(k) \leq \bar{h}_{i}, \\
\left|w_{i}(k)\right| \leq \bar{w}_{i}=\max \left\{h_{i r} w_{i r}, h_{i l} w_{i l}\right\} .
\end{gathered}
$$

Assumption 2 (see [29-31]). $g_{i}(\xi(k)), i \in P$, are strictly positive or negative and the signs of $g_{i}(\xi(k))$ are known. Without loss of generality, we suppose that $g_{i}(\xi(k))$ are positive with $\underline{g}_{i}$ and $\bar{g}_{i}$ being the lower bound and upper bound, respectively. It implies that $\bar{g}_{i} \geq \underline{g}_{i}>0$.

The control objective of this paper is to design dead zone inputs $v_{i}(k)$ of subsystems, such that

(1) the system state $\xi(k)$ can track a given signal $\xi^{d}(k)=$ $\left[\xi_{1}^{d}(k), \xi_{2}^{d}(k), \ldots, \xi_{n}^{d}(k)\right]^{T}$, or equivalently speaking, the output can track a desired trajectory $y^{d}(k)$,

(2) all the signals in the closed-loop system are bounded under arbitrary switching signals.

Assumption 3 (see [32]). The desired trajectory of the system state $\xi^{d}(k)=\left[\xi_{1}^{d}(k), \xi_{2}^{d}(k), \ldots, \xi_{n}^{d}(k)\right]^{T} \in \mathfrak{R}^{n}$ satisfies the condition $\xi_{j}^{d}(k+1)=\xi_{j+1}^{d}(k), j=1, \ldots, n-1$. Moreover $y^{d}(k)$ is a known smooth bounded function.

According to Assumption 2 and system (1), we can obtain that $\xi_{j}^{d}(k)=y^{d}(k+j-1), j=1, \ldots, n$.

\section{Neural Networks}

Similar to FLSs, NNs also have the approximation properties. In this paper, we consider the case that there exist unknown functions in the systems. Here, we use RBFNNs to approximate the unknown functions of subsystems.

For any unknown continuous function $F(X)$ defined on $\Omega \subset \mathfrak{R}^{n}$, there exists $\mathrm{NN} \theta^{T} \varphi(X)$, such that

$$
F(X)=\theta^{T} \varphi(X)+\varepsilon(X),
$$

where $X \in \Omega \subset \Re^{n}$ is the input variable of the $\mathrm{NN}$, $\theta=\left[\theta_{1}, \ldots, \theta_{\ell}\right]^{T}$ is the weight vector with $\ell$ being the node number of NNs, $\varepsilon(X)$ is the approximation error, and $\varphi(X)=$ 
$\left[\phi_{1}(X), \ldots, \phi_{\ell}(X)\right]^{T}$ is the basis function. In RBFNNs, $\phi_{i}(X)$, $i=1, \ldots, \ell$, are chosen as Gaussian functions

$$
\phi_{i}(X)=\exp \left(-\frac{\left(X-c_{i}\right)^{T}\left(X-c_{i}\right)}{\mu_{i}^{2}}\right),
$$

where $c_{i}=\left[c_{i 1}, \ldots, c_{i n}\right]^{T}$ and $\mu_{i}, i=1, \ldots, \ell$, are the centers and widths of the Gaussian functions, respectively.

According to (7), we have $0<\phi_{i}(X) \leq 1$. Thus, we can further obtain that $\phi_{i}^{2}(X) \leq 1, i=1, \ldots, \ell$. This leads to the following inequality:

$$
\varphi^{T}(X) \varphi(X) \leq \ell .
$$

In this paper, since there are unknown functions in (11), we cannot obtain $v_{i}^{0}, i=1, \ldots, n$, directly. Thus, the neural networks are used to approximate the unknown function $v_{i}^{0}$, $i=1, \ldots, n$. In fact, the neural networks in this paper are used for identification.

\section{Dead Zone Input Design}

Define the tracking error at instant $k$ as

$$
e_{j}(k)=\xi_{j}(k)-\xi_{j}^{d}(k+1)=\xi_{j}(k)-y^{d}(k+j-1) .
$$

Then, we can get the $n$th state error $e_{n}(k)=\xi_{n}(k)-y^{d}(k+n-1)$. Furthermore, we have

$$
\begin{aligned}
e_{n}(k+1)= & f_{i}(\xi(k))+g_{i}(\xi(k)) u_{i}(k)-y^{d}(k+n) \\
= & f_{i}(k)+g_{i}(k) h_{i}(k) v_{i}(k)+g_{i}(k) w_{i}(k) \\
& -y^{d}(k+n),
\end{aligned}
$$

where $f_{i}(k)$ and $g_{i}(k)$ are the abbreviations of $f_{i}(\xi(k))$ and $g_{i}(\xi(k))$, respectively.

Define functions

$$
v_{i}^{0}(k)=-\frac{1}{g_{i}(k) m_{i}(k)}\left(f_{i}(k)-y^{d}(k+n)\right), \quad i \in P .
$$

Because of the existence of the unknown functions in (11), $v_{i}^{0}(k)$ cannot be directly obtained. Here, we use RBFNNs to approximate them

$$
v_{i}^{0}(k)=\theta_{i}^{T} \varphi(S(k))+\varepsilon_{i}(S(k)), \quad i \in P,
$$

where $\theta_{i}$ are the weight matrices which are assumed to be bounded, that is, $\left\|\theta_{i}\right\| \leq \bar{\theta}_{i}$, and $\varphi(S(k))$ and $\varepsilon_{i}(S(k))$ are the basis function vector and the approximation errors, respectively. Furthermore, $\|\varphi(S(k))\|^{2} \leq \ell$ and $\left\|\varepsilon_{i}(S(k))\right\| \leq \bar{\varepsilon}_{i}$. $S(k)=\left[\xi^{T}(k), y^{d T}(k), y^{d T}(k+n)\right]^{T}$ is the input vector of RBFNNs.

By adding and subtracting $g_{i}(k) h_{i}(k) v_{i}^{0}(k)$ on the right side of equation (10), we can conclude that

$$
\begin{aligned}
& e_{n}(k+1) \\
& \quad=g_{i}(k) h_{i}(k)\left[v_{i}(k)-\theta_{i}^{T} \varphi(k)-\varepsilon_{i}(k)+\frac{w_{i}(k)}{h_{i}(k)}\right],
\end{aligned}
$$

where $\varphi(k)$ and $\varepsilon_{i}(k)$ are the shorthand for $\varphi(S(k))$ and $\varepsilon_{i}^{*}(S(k))$, respectively. Denote that $w_{i}(k) / h_{i}(k) \leq \bar{w}_{i} / \underline{h}_{i} \triangleq \eta_{i}$ and $\eta_{i}, i \in P$, are unknown constants. Let $\widehat{\eta}_{i}$ be the estimation of $\eta_{i}$ and lwt $\widetilde{\eta}_{i}(k)=\widehat{\eta}_{i}(k)-\eta_{i}$ be the estimation error.

According to (13), we design the controllers as

$$
v_{i}(k)=\widehat{\theta}_{i}^{T}(k) \varphi(k)+\widehat{\eta}_{i}(k),
$$

where $\widehat{\theta}_{i}(k)$ are the estimation of $\theta_{i}(k)$. Let the estimation error satisfy $\widetilde{\theta}_{i}(k)=\widehat{\theta}_{i}(k)-\theta_{i}$.

The switched update laws are designed as

$$
\begin{aligned}
& \widehat{\theta}_{i}(k+1) \begin{cases}\widehat{\theta}_{i}(k)-\Gamma_{i}\left[\delta_{i} \widehat{\theta}_{i}(k)+\varphi(k) e_{n}(k+1)\right], & \sigma=i, \\
0, & \sigma \neq i,\end{cases} \\
& \widehat{\eta}_{i}(k+1) \\
& \quad= \begin{cases}\widehat{\eta}_{i}(k)-\mu_{i}\left[\tau_{i} \widehat{\eta}_{i}(k)+e_{n}(k+1)\right], & \sigma=i, \\
0, & \sigma \neq i,\end{cases}
\end{aligned}
$$

where $\Gamma_{i}$ and $\mu_{i}$ are positive matrices and positive design parameters, respectively.

Based on (14), (13) becomes

$$
\begin{aligned}
e_{n}(k+1)=g_{i}(k) h_{i}(k) \\
\cdot\left[\widetilde{\theta}_{i}^{T} \varphi(k)-\varepsilon_{i}(k)+\widehat{\eta}_{i}(k)+\frac{w_{i}(k)}{h_{i}(k)}\right]=g_{i}(k) h_{i}(k) \\
\cdot\left[\widetilde{\theta}_{i}^{T} \varphi(k)+\widetilde{\eta}_{i}(k)-\varepsilon_{i}(k)+\eta_{i}+\frac{w_{i}(k)}{h_{i}(k)}\right] .
\end{aligned}
$$

\section{Stability Analysis}

In this section, the stability of switched system (1) and the boundedness of all the signals in the closed-loop system are proved under arbitrary switching signals.

Theorem 4. Consider the discrete-time switched system (1), the dead zone (3), the switched update laws given by (15), and the input of the ith dead zone selected as in (14) under Assumptions 1-3. If the design parameters satisfy the conditions

$$
\begin{aligned}
& \gamma_{\sigma}<\frac{1-\mu_{\sigma} \bar{g} \bar{h}}{3+l \bar{g} \bar{h}+l+\mu_{\sigma}}, \\
& \delta_{\sigma}<\frac{1}{\gamma_{\sigma}+\gamma_{\sigma} \bar{g} \bar{h}}, \\
& \mu_{\sigma}<\frac{\gamma_{\sigma}}{\gamma_{\sigma}+\bar{g} \bar{h} \tau_{\sigma}},
\end{aligned}
$$

then the boundedness of all the signals in the closed-loop switched system can be ensured and the state $\xi(k)$ can track the reference signal $\xi^{d}(k)$ and the system output can follow the desired trajectory $y^{d}(k)$. 
Proof. Choose the following common Lyapunov function for system (1) as

$$
\begin{aligned}
V(k)= & \frac{1}{\bar{g} \bar{h}} e_{n}^{2}(k)+\sum_{i=1}^{p} \operatorname{tr}\left\{\widetilde{\theta}_{i}^{T}(k) \Gamma_{i}^{-1} \widetilde{\theta}_{i}(k)\right\} \\
& +\sum_{i=1}^{p} \frac{1}{\mu_{i}} \widetilde{\eta}_{i}^{2}(k), \quad i \in P,
\end{aligned}
$$

where $\bar{g}=\max _{i \in P} \bar{g}_{i}$ and $\bar{h}=\max _{i \in P} \bar{h}_{i}$.

The first difference of $V(k)$ is

$$
\begin{aligned}
\Delta V(k)= & \frac{1}{\bar{g} \bar{h}}\left[e_{n}^{2}(k+1)-e_{n}^{2}(k)\right] \\
& +\sum_{i=1}^{p} \operatorname{tr}\left\{\widetilde{\theta}_{i}^{T}(k+1) \Gamma_{i}^{-1} \widetilde{\theta}_{i}(k+1)\right\} \\
& -\sum_{i=1}^{p} \operatorname{tr}\left\{\widetilde{\theta}_{i}^{T}(k) \Gamma_{i}^{-1} \widetilde{\theta}_{i}(k)\right\}+\sum_{i=1}^{p} \frac{1}{\mu_{i}} \widetilde{\eta}_{i}^{2}(k+1) \\
& -\sum_{i=1}^{p} \frac{1}{\mu_{i}} \widetilde{\eta}_{i}^{2}(k) .
\end{aligned}
$$

Substituting (15) into (19), one has

$$
\begin{aligned}
& \Delta V(k)=\frac{1}{\bar{g} \bar{h}}\left[e_{n}^{2}(k+1)-e_{n}^{2}(k)\right]-2 \operatorname{tr}\left\{\widetilde{\theta}_{\sigma}^{T}(k)\right. \\
& \left.\cdot\left[\delta_{\sigma} \widehat{\theta}_{\sigma}(k)+\varphi(k) e_{n}(k+1)\right]\right\} \\
& +\operatorname{tr}\left\{\left[\delta_{\sigma} \widehat{\theta}_{\sigma}(k)+\varphi(k) e_{n}(k+1)\right]^{T}\right. \\
& \left.\cdot \Gamma_{\sigma}\left[\delta_{\sigma} \widehat{\theta}_{\sigma}(k)+\varphi(k) e_{n}(k+1)\right]\right\}-2 \widetilde{\eta}_{\sigma}(k) \\
& \cdot\left[\tau_{\sigma} \widehat{\eta}_{\sigma}(k)+e_{n}(k+1)\right]+\mu_{\sigma}\left[\tau_{\sigma} \widehat{\eta}_{\sigma}(k)+e_{n}(k+1)\right]^{2} \\
& =\frac{1}{\bar{g} \bar{h}}\left[e_{n}^{2}(k+1)-e_{n}^{2}(k)\right]-2 \delta_{\sigma} \widetilde{\theta}_{\sigma}^{T}(k) \hat{\theta}_{\sigma}(k) \\
& -2 \widetilde{\theta}_{\sigma}^{T}(k) \varphi(k) e_{n}(k+1)+\operatorname{tr}\left\{\delta_{\sigma}^{2} \widehat{\theta}_{\sigma}^{T}(k) \Gamma_{\sigma} \widehat{\theta}_{\sigma}(k)\right\} \\
& +\operatorname{tr}\left\{e_{n}(k+1) \varphi^{T}(k) \Gamma_{\sigma} \varphi(k) e_{n}(k+1)\right\}+2 \\
& +\operatorname{tr}\left\{\delta_{\sigma} \widehat{\theta}_{\sigma}^{T}(k) \Gamma_{\sigma} \varphi(k) e_{n}(k+1)\right\}-2 \tau_{\sigma} \widetilde{\eta}_{\sigma}(k) \widehat{\eta}_{\sigma}(k) \\
& +\mu_{\sigma} \tau_{\sigma} \widehat{\eta}_{\sigma}^{2}(k)-2 \widetilde{\eta}_{\sigma}(k) e_{n}(k+1)+\mu_{\sigma} e_{n}^{2}(k+1) \\
& +2 \mu_{\sigma} \tau_{\sigma} \widehat{\eta}_{\sigma}(k) e_{n}(k+1),
\end{aligned}
$$

with $\gamma_{\sigma}$ being the maximum eigenvalue of $\Gamma_{\sigma}$.

According to (16), we can conclude that

$$
\begin{aligned}
\widetilde{\theta}_{i}^{T}(k) \varphi(k)+\widetilde{\eta}_{i}(k)= & \frac{e_{n}(k+1)}{g_{i}(k) h_{i}(k)}+\varepsilon_{i}(k)-\eta_{i} \\
& -\frac{w_{i}(k)}{h_{i}(k)} .
\end{aligned}
$$

Then, $\Delta V(k)$ becomes

$$
\begin{aligned}
\Delta V(k) \leq & \frac{1}{\bar{g} \bar{h}}\left[e_{n}^{2}(k+1)-e_{n}^{2}(k)\right] \\
& -\frac{2}{\bar{g}_{\sigma}(k) \bar{h}_{\sigma}(k)} e_{n}^{2}(k+1) \\
& -2 \varepsilon_{\sigma}(k) e_{n}(k+1)+2 \eta_{\sigma} e_{n}(k+1) \\
& +\frac{w_{\sigma}(k)}{h_{\sigma}(k)} e_{n}(k+1)-2 \delta_{\sigma} \widetilde{\theta}_{\sigma}^{T}(k) \widehat{\theta}_{\sigma}(k) \\
& +\mu_{\sigma} \tau_{\sigma} \widehat{\eta}_{\sigma}^{2}(k)+\gamma_{\sigma} \delta_{\sigma}^{2}\left\|\widehat{\theta}_{\sigma}(k)\right\|^{2} \\
& +\gamma_{\sigma} l e_{n}^{2}(k+1) \\
& +2 \gamma_{\sigma} \delta_{\sigma} \widehat{\theta}_{\sigma}^{T}(k) \varphi(k) e_{n}(k+1) \\
& -2 \tau_{\sigma} \widetilde{\eta}_{\sigma}(k) \widehat{\eta}_{\sigma}(k)+\mu_{\sigma} e_{n}^{2}(k+1) \\
& +2 \mu_{\sigma} \tau_{\sigma} \widehat{\eta}_{\sigma}(k) e_{n}(k+1) .
\end{aligned}
$$

Based on Assumption 2, it holds that

$$
-\frac{2}{g_{i}(k) h_{i}(k)} e_{n}^{2}(k+1) \leq-\frac{2}{\bar{g} \bar{h}} e_{n}^{2}(k+1) .
$$

By using Young's inequality, we have the following inequalities:

$$
\begin{aligned}
& -2 \varepsilon_{\sigma}(k) e_{n}(k+1) \leq \frac{\bar{g} \bar{h}}{\gamma_{\sigma}} \bar{\varepsilon}_{\sigma}^{2}+\frac{\gamma_{\sigma}}{\bar{g} \bar{h}} e_{n}^{2}(k+1), \\
& 2 \eta_{\sigma} e_{n}(k+1) \leq \frac{\bar{g} \bar{h}}{\gamma_{\sigma}} \eta_{\sigma}^{2}+\frac{\gamma_{\sigma}}{\bar{g} \bar{h}} e_{n}^{2}(k+1), \\
& 2 \frac{w_{i}(k)}{h_{i}(k)} e_{n}(k+1) \leq \frac{\bar{g} \bar{h}}{\gamma_{\sigma}} \eta_{i}^{2}+\frac{\gamma_{\sigma}}{\bar{g} \bar{h}} e_{n}^{2}(k+1), \\
& 2 \gamma_{\sigma} \delta_{\sigma} \widehat{\theta}_{\sigma}^{T}(k) \varphi(k) e_{n}(k+1) \\
& \leq \bar{g} \bar{h} \gamma_{\sigma} \delta_{\sigma}^{2}\left\|\hat{\theta}_{\sigma}(k)\right\|^{2}+\frac{\gamma_{\sigma} l}{\bar{g} \bar{h}} e_{n}^{2}(k+1), \\
& 2 \mu_{\sigma} \tau_{\sigma} \hat{\eta}_{\sigma}(k) e_{n}(k+1) \\
& \leq \frac{\bar{g} \bar{h} \mu_{\sigma} \tau_{\sigma}^{2}}{\gamma_{\sigma}} \widehat{\eta}_{\sigma}^{2}(k)+\frac{\gamma_{\sigma} \mu_{\sigma}}{\bar{g} \bar{h}} e_{n}^{2}(k+1),
\end{aligned}
$$

and we consider the following equations:

$$
\begin{aligned}
& 2 \widetilde{\theta}_{\sigma}^{T}(k) \hat{\theta}_{\sigma}(k)=\left\|\tilde{\theta}_{\sigma}(k)\right\|^{2}+\left\|\hat{\theta}_{\sigma}(k)\right\|^{2}-\left\|\theta_{\sigma}\right\|^{2}, \\
& 2 \widetilde{\eta}_{\sigma}(k) \hat{\eta}_{\sigma}(k)=\widetilde{\eta}_{\sigma}^{2}(k)+\widehat{\eta}_{\sigma}^{2}(k)-\eta_{\sigma}^{2} .
\end{aligned}
$$


Then, we have

$$
\begin{aligned}
\Delta V(k) \leq & \frac{1}{\bar{g} \bar{h}}\left[e_{n}^{2}(k+1)-e_{n}^{2}(k)\right]-\frac{2}{\bar{g} \bar{h}} e_{n}^{2}(k+1) \\
& +\frac{\bar{g} \bar{h}}{\gamma_{\sigma}} \bar{\varepsilon}_{\sigma}^{2}+\frac{\gamma_{\sigma}}{\bar{g} \bar{h}} e_{n}^{2}(k+1)+\frac{2 \bar{g} \bar{h}}{\gamma_{\sigma}} \eta_{\sigma}^{2} \\
& +\frac{2 \gamma_{\sigma}}{\bar{g} \bar{h}} e_{n}^{2}(k+1)-\delta_{\sigma}\left\|\widetilde{\theta}_{\sigma}(k)\right\|^{2} \\
& -\delta_{\sigma}\left\|\widehat{\theta}_{\sigma}(k)\right\|^{2}+\delta_{\sigma}\left\|\theta_{\sigma}\right\|^{2}+\mu_{\sigma} \tau_{\sigma} \widehat{\eta}_{\sigma}^{2}(k) \\
& +\gamma_{\sigma} \delta_{\sigma}^{2}\left\|\hat{\theta}_{\sigma}(k)\right\|^{2}+\gamma_{\sigma} l e_{n}^{2}(k+1) \\
& +\bar{g} \bar{h} \gamma_{\sigma} \delta_{\sigma}^{2}\left\|\hat{\theta}_{\sigma}(k)\right\|^{2}+\frac{\gamma_{\sigma} l}{\bar{g} \bar{h}} e_{n}^{2}(k+1) \\
& -\tau_{\sigma} \widetilde{\eta}_{\sigma}^{2}(k)-\tau_{\sigma} \hat{\eta}_{\sigma}^{2}(k)+\tau_{\sigma} \eta_{\sigma}^{2} \\
& +\mu_{\sigma} e_{n}^{2}(k+1) \frac{\bar{g} \bar{h} \mu_{\sigma} \tau_{\sigma}^{2}}{\gamma_{\sigma}} \hat{\eta}_{\sigma}^{2}(k) \\
& +\frac{\gamma_{\sigma} \mu_{\sigma}}{\bar{g} \bar{h}} e_{n}^{2}(k+1) \\
\leq & -\frac{1}{\bar{g} \bar{h}} M_{1} e_{n}^{2}(k+1)-\delta_{\sigma} M_{2}\left\|\hat{\theta}_{\sigma}(k)\right\|^{2} \\
& -\tau_{\sigma} M_{3} \hat{\eta}_{\sigma}^{2}(k)-\frac{1}{\bar{g} \bar{h}} e_{n}^{2}(k)-\delta_{\sigma}\left\|\tilde{\theta}_{\sigma}(k)\right\|^{2} \\
& -\tau_{\sigma} \widetilde{\eta}_{\sigma}^{2}(k)+\beta,
\end{aligned}
$$

where

$$
\begin{aligned}
& M_{1}=1-3 \gamma_{\sigma}-\gamma_{\sigma} l \bar{g} \bar{h}-\gamma_{\sigma} l-\mu_{\sigma} \bar{g} \bar{h}-\gamma_{\sigma} \mu_{\sigma}, \\
& M_{2}=1-\gamma_{\sigma} \delta_{\sigma}-\gamma_{\sigma} \delta_{\sigma} \bar{g} \bar{h}, \\
& M_{3}=1-\mu_{\sigma}-\frac{\bar{g} \bar{h} \mu_{\sigma} \tau_{\sigma}}{\gamma_{\sigma}}, \\
& \beta=\frac{\bar{g} \bar{h}}{\gamma_{\sigma}} \bar{\varepsilon}_{\sigma}^{2}+\frac{2 \bar{g} \bar{h}}{\gamma_{\sigma}} \eta_{\sigma}^{2}+\delta_{\sigma}\left\|\theta_{\sigma}\right\|^{2}+\tau_{\sigma} \eta_{\sigma}^{2} .
\end{aligned}
$$

If the design parameters satisfy condition (17), then we can deduce that

$$
\Delta V_{i}(k) \leq-\frac{1}{\bar{g} h} e_{n}^{2}(k)-\delta_{\sigma}\left\|\widetilde{\theta}_{\sigma}(k)\right\|^{2}-\tau_{\sigma} \widetilde{\eta}_{\sigma}^{2}(k)+\beta .
$$

If $\left|e_{n}(k)\right|>\sqrt{\bar{g} \bar{h} \beta}$ or $\left\|\widetilde{\theta}_{\sigma}(k)\right\|>\sqrt{\beta / \delta_{\sigma}}$ or $\left|\widetilde{\eta}_{\sigma}(k)\right|>$ $\sqrt{\beta / \tau_{\sigma}}$ holds, we have $\Delta V_{i}(k)<0$. According to the standard Lyapunov extension theorem, the tracking error $e_{n}(k)$ and the estimation errors $\widetilde{\theta}_{\sigma}(k)$ and $\widetilde{\eta}_{\sigma}(k)$ are bounded. Since $e_{j}(k)=\xi_{j}(k)-\xi_{j}^{d}(k+1)=e_{n}(k-n+j), j=1, \ldots, n$, we can easily know that $e_{j}(k), j=1, \ldots, n-1$, are also bounded. Furthermore, because $\theta_{\sigma}$ and $\eta_{\sigma}$ are bounded, the boundedness of the estimated parameters $\widehat{\theta}_{\sigma}(k)$ and $\widehat{\eta}_{\sigma}(k)$ is guaranteed by $\widetilde{\theta}_{\sigma}(k)=\widehat{\theta}_{\sigma}(k)-\theta_{\sigma}$ and $\widetilde{\eta}_{\sigma}(k)=\widehat{\eta}_{\sigma}(k)-\eta_{\sigma}$. Thus, from (14), we know that the dead zone inputs $v_{\sigma}$ are bounded. Based on the definition of dead zone, one can conclude that the control inputs $u_{\sigma}$ are also bounded. Therefore, all the signals in the closed-loop system are bounded under arbitrary switching signals.

Remark 5. As we all know that a switched system might be unstable even if all the subsystems are stable, arbitrary switching method can guarantee the stability of the switched system in this case. A sufficient condition for analysing the stability of switched systems under arbitrary switching signals is that there exists a common Lyapunov function. In many cases, it is easy to find a Lyapunov function for each subsystem. However, a common Lyapunov is hard to find for switched systems. In this paper, we have successfully found a common Lyapunov function shown as in (18).

Remark 6. There are many parameters in the design procedure. $h_{i r}, h_{i l}, w_{i r}$, and $w_{i l}, i=1,2$, are parameters of dead zone. They are all positive based on Assumption 1. In addition, $\Gamma_{i}, \delta_{i}, \mu_{i}$, and $\tau_{i}, i=1,2$, are introduced to improve the flexibility of the update laws. In this paper, they are chosen to be positive. But how to choose a set of optimal ones to ensure the satisfactory performance is still an open problem; they need to be flexibly selected. In the following, a constructive suggestion on how to choose these parameters is given. From the above stability analysis, we can see that the bounds of $e_{n}(k), \tilde{\theta}_{\sigma}(k)$, and $\widetilde{\eta}_{\sigma}(k)$ depend on the values of $\bar{g} \bar{h}, \beta, \delta_{\sigma}(k)$, and $\tau_{\sigma}(k)$. Actually, we can select smaller $\bar{g} \bar{h}$ and larger $\delta_{\sigma}(k)$ and $\tau_{\sigma}(k)$ to obtain smaller bounds of $e_{n}(k), \widetilde{\theta}_{\sigma}(k)$, and $\tilde{\eta}_{\sigma}(k)$ to further get good performance. But, it is important to note that these parameters must satisfy conditions (17). In the simulation, a trial-and-error method is used.

\section{Simulation}

In this section, we apply the proposed approach to the following discrete-time switched nonlinear system:

$$
\begin{aligned}
\xi_{1}(k+1) & =\xi_{2}(k), \\
\xi_{2}(k+1) & =f_{\sigma}^{1}(\xi(k))+g_{\sigma}^{1}(\xi(k)) u_{\sigma}(k), \\
\xi_{3}(k+1) & =\xi_{4}(k), \\
\xi_{4}(k+1) & =f_{\sigma}^{2}(\xi(k))+g_{\sigma}^{2}(\xi(k)) u_{\sigma}(k), \\
y_{1}(k) & =\xi_{1}(k), \\
y_{2}(k) & =\xi_{3}(k),
\end{aligned}
$$

where $f_{1}^{1}(\bar{\xi}(k))=\xi_{1}(k)+\xi_{2}^{2}(k), f_{1}^{2}(\bar{\xi}(k))=1 / 2\left(\xi_{1}(k)+\xi_{2}\right)(k)$, $g_{1}^{1}(\bar{\xi}(k))=\xi_{1}(k) \xi_{2}(k), g_{1}^{2}(\bar{\xi}(k))=\xi_{1}(k) \xi_{2}(k)+\xi_{1}(k) \xi_{2}^{2}(k)$, $f_{2}^{1}(\bar{\xi}(k))=\xi_{3}(k)+\xi_{1}^{2}(k), f_{2}^{2}(\bar{\xi}(k))=\xi_{1}(k)+\xi_{3}(k), g_{2}^{1}(\bar{\xi}(k))=$ $\xi_{1}(k) \xi_{3}^{2}(k)+\xi_{3}(k) \xi_{4}^{2}(k)$, and $g_{2}^{2}(\bar{\xi}(k))=\xi_{1}(k) \xi_{3}(k)+\xi_{3}(k) \xi_{4}(k)$. The switching signal takes values in $P=\{1,2\}$. 
The dead zone is described as

$$
\begin{aligned}
u_{i}(k) & =D\left(v_{i}(k)\right) \\
& = \begin{cases}h_{i r}\left(v_{i}(k)-w_{i r}\right), & \text { if } v_{i}(k) \geq w_{i r}, \\
0, & \text { if }-w_{i l}<v_{i}(k)<w_{i r}, \\
h_{i l}\left(v_{i}(k)+w_{i l}\right), & \text { if } v_{i}(k) \leq-w_{i l} .\end{cases}
\end{aligned}
$$

The parameters of the dead zone inputs are selected as $h_{1 r}=0.3, h_{2 r}=0.4, h_{1 l}=0.2, h_{2 l}=0.3, w_{1 r}=0.5, w_{2 r}=0.7$, $w_{1 l}=0.6$, and $w_{2 l}=0.8$.

Define the tracking errors $e_{1}(k)=y_{1}(k)-y_{d_{1}}(k)=$ $\xi_{1}(k)-y_{d_{1}}(k)$ and $e_{2}(k)=y_{2}(k)-y_{d_{2}}(k)=\xi_{3}(k)-y_{d_{2}}(k)$. The desired signals $y_{d_{1}}(k)$ and $y_{d_{2}}(k)$ are selected as $y_{d_{1}}(k)=$ $0.28[\sin (k \pi / 10)+\cos (k \pi / 10)]$ and $y_{d_{2}}(k)=0.22[\sin (k \pi / 10)+$ $\cos (k \pi / 10)]$, respectively. The initial values for the system states are $\xi_{1}(0)=0.01, \xi_{2}(0)=0.01, \xi_{1}(0)=0.01$, and $\xi_{2}(0)=0.01$.

The dead zone input is designed for each subsystem

$$
v_{i}(k)=\widehat{\theta}_{i}^{T}(k) \varphi(k)+\widehat{\eta}_{i}(k),
$$

where $\varphi(k)$ is the Gaussian function.

The switched update laws are designed for $i=1,2$, as

$$
\begin{aligned}
& \widehat{\theta}_{i}(k+1) \\
& \quad= \begin{cases}\widehat{\theta}_{i}(k)-\Gamma_{i}\left[\delta_{i} \widehat{\theta}_{i}(k)+\varphi(k) e_{n}(k+1)\right], & \sigma=i, \\
0, & \sigma \neq i,\end{cases}
\end{aligned}
$$$$
\widehat{\eta}_{i}(k+1)
$$

$$
= \begin{cases}\widehat{\eta}_{i}(k)-\mu_{i}\left[\tau_{i} \widehat{\eta}_{i}(k)+e_{n}(k+1)\right], & \sigma=i, \\ 0, & \sigma \neq i,\end{cases}
$$

where $\Gamma_{1}=0.5 I, \Gamma_{2}=0.2 I, \delta_{1}=2, \delta_{2}=2.5, \mu_{1}=0.5, \mu_{2}=0.7$, $\tau_{1}=2.3$, and $\tau_{2}=1.8$.

Furthermore, we give the simulation results in Figures $1-7$. Figure 1 gives the curves of $y_{1}$ and $y_{d_{1}}$. Figure 2 shows the trajectories of $y_{2}$ and $y_{d_{2}}$. Figures 3 and 4 display the tracking errors $e_{1}$ and $e_{2}$, respectively. From Figures 1-4, we can observe that a good tracking performance is obtained. Moreover, the dead zone inputs are shown in Figures 5 and 6 , which explain the boundedness of the dead zone inputs. Figure 7 is the switching signal.

\section{Conclusion}

We design a controller of each subsystem for a class of discrete-time switched nonlinear systems with dead zone inputs under arbitrary switching signals in this paper. The unknown functions are approximated by RBFNNs. Switched update laws are designed while the parameter estimation is invariable until its corresponding subsystem is active. Then, the closed-loop system is stable and all the signals are bounded. Due to the complicated framework of the discretetime switched nonlinear systems and the existence of the dead zone, it brings about great difficulties for controlling such a class of systems.

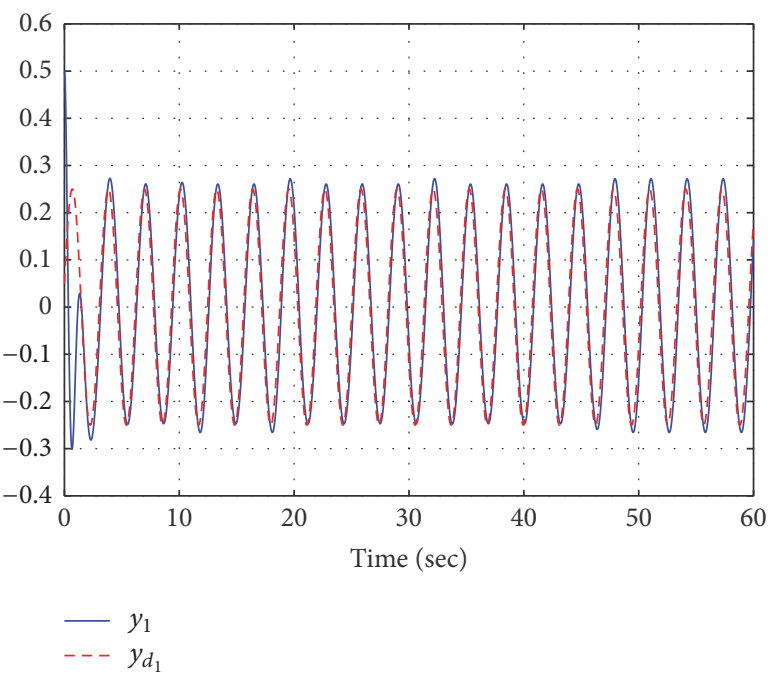

FIGURE 1: The trajectories of $y_{1}$ and $y_{d_{1}}$.

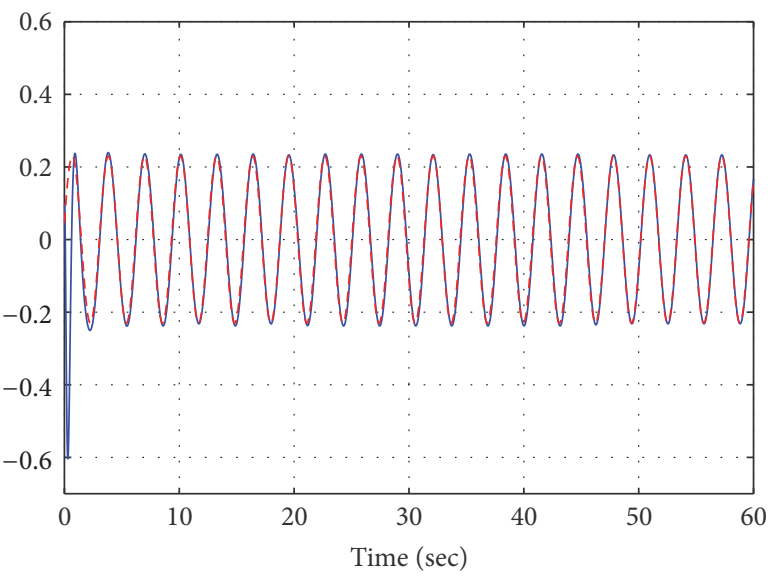

$y_{2}$
$---y_{d_{2}}$

FIGURE 2: The trajectories of $y_{2}$ and $y_{d_{2}}$.

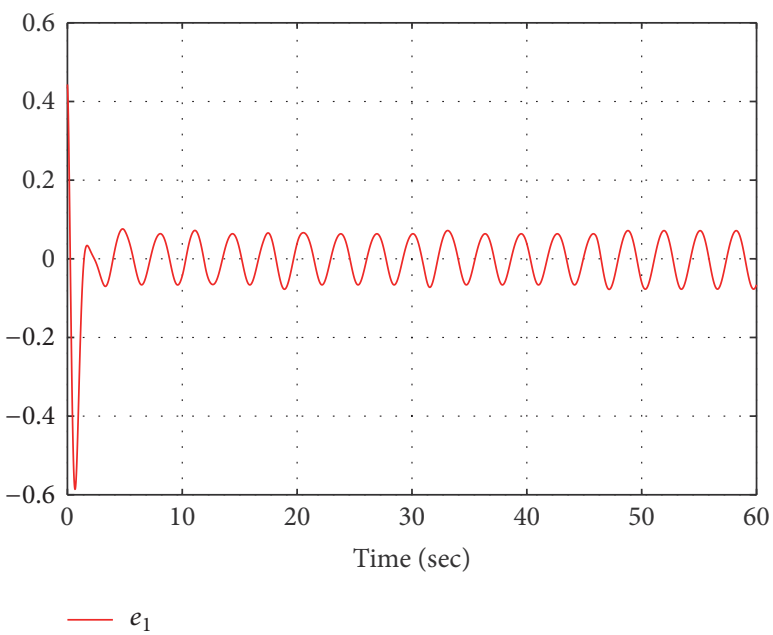

FIGURE 3: The trajectory of tracking error $e_{1}$. 


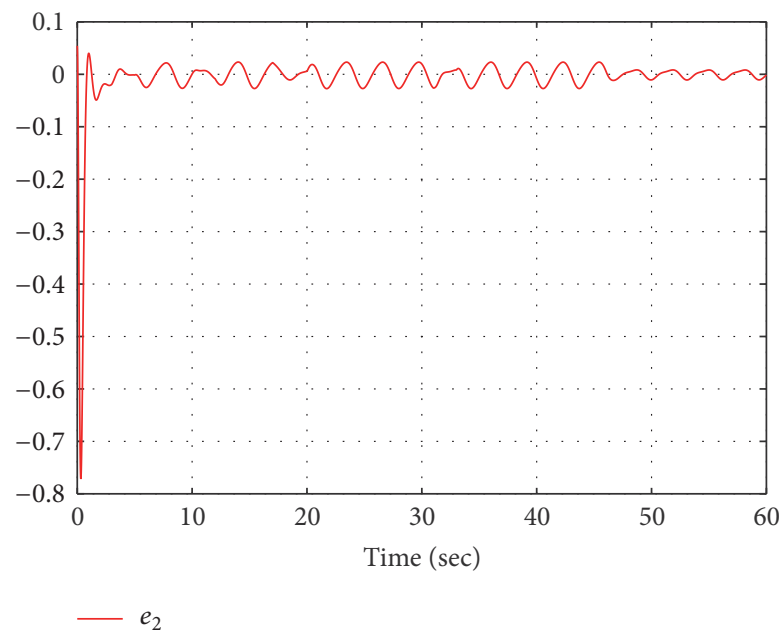

FIgURE 4: The trajectory of tracking error $e_{2}$.

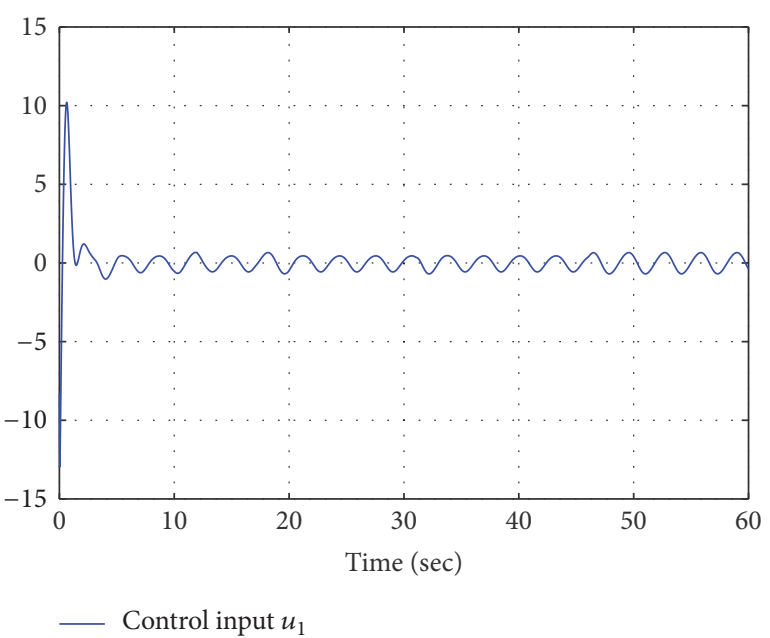

FIgURE 5: Control input $u 1$.

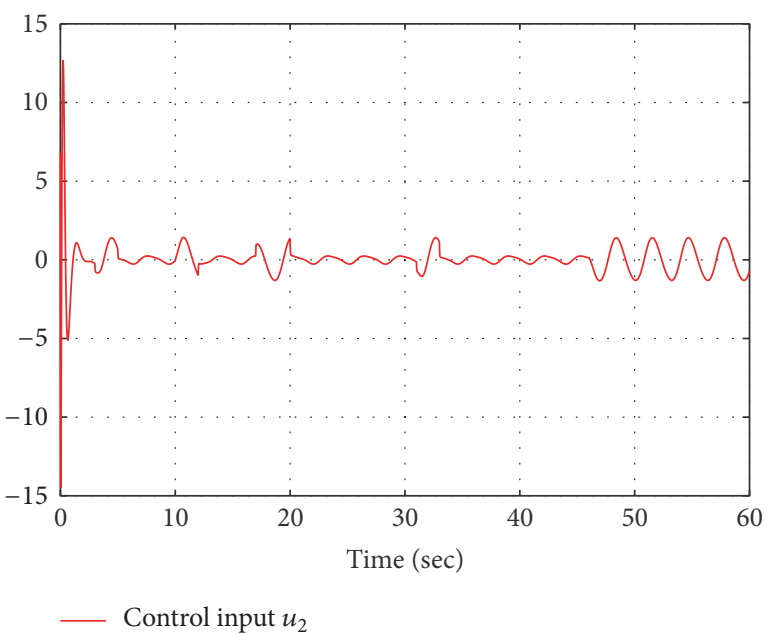

Figure 6: Control input $u 2$.

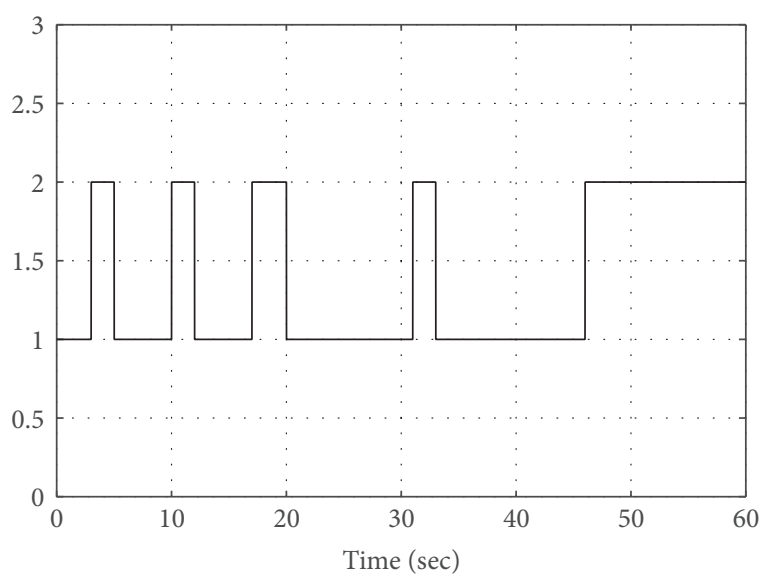

FIGURE 7: Switching signal $\sigma$.

\section{Conflicts of Interest}

The authors declare that there are no conflicts of interest regarding the publication of this paper.

\section{Acknowledgments}

This work was supported by the Science and Technology Key Projects of Henan Province (162102210080).

\section{References}

[1] R. R. Selmic and F. L. Lewis, "Neural-network approximation of piecewise continuous functions: application to friction compensation," IEEE Transactions on Neural Networks, vol. 13, no. 3, pp. 745-751, 2002.

[2] L. Liu, Z. S. Wang, Z. J. Huang, and H. G. Zhang, "Adaptive predefined performance control for MIMO systems with unknown direction via generalized fuzzy hyperbolic model," IEEE Transactions on Fuzzy Systems, 2016.

[3] R. Yuan, X. Tan, G. Fan, and J. Yi, "Robust adaptive neural network control for a class of uncertain nonlinear systems with actuator amplitude and rate saturations," Neurocomputing, vol. 125, pp. 72-80, 2014.

[4] D. Liu, D. Wang, D. Zhao, Q. Wei, and N. Jin, "Neural-networkbased optimal control for a class of unknown discrete-time nonlinear systems using globalized dual heuristic programming," IEEE Transactions on Automation Science and Engineering, vol. 9, no. 3, pp. 628-634, 2012.

[5] C.-H. Lin, "Hybrid recurrent wavelet neural network control of PMSM servo-drive system for electric scooter," International Journal of Control, Automation and Systems, vol. 12, no. 1, pp. 177-187, 2014.

[6] R.-J. Wai, M.-W. Chen, and Y.-K. Liu, "Design of adaptive control and fuzzy neural network control for single-stage boost inverter," IEEE Transactions on Industrial Electronics, vol. 62, no. 9, pp. 5434-5445, 2015.

[7] W. He, C. Sun, and S. S. Ge, "Top tension control of a flexible marine riser by using integral-barrier lyapunov function," IEEE/ASME Transactions on Mechatronics, vol. 20, no. 2, pp. 497-505, 2015. 
[8] Z. Liu, F. Wang, Y. Zhang, X. Chen, and C. L. P. Chen, "Adaptive tracking control for a class of nonlinear systems with a fuzzy dead-zone input," IEEE Transactions on Fuzzy Systems, vol. 23, no. 1, pp. 193-204, 2015.

[9] Y.-J. Liu, L. Tang, S. Tong, and C. L. P. Chen, "Adaptive NN controller design for a class of nonlinear MIMO discrete-time systems," IEEE Transactions on Neural Networks and Learning Systems, vol. 26, no. 5, pp. 1007-1018, 2015.

[10] H. Zhang, R. Song, Q. Wei, and T. Zhang, "Optimal tracking control for a class of nonlinear discrete-time systems with time delays based on heuristic dynamic programming," IEEE Transactions on Neural Networks, vol. 22, no. 12, pp. 1851-1862, 2011.

[11] L. Liu, Z. Wang, and H. Zhang, "Adaptive fault-tolerant tracking control for MIMO discrete-time systems via reinforcement learning algorithm with less learning parameters," IEEE Transactions on Automation Science and Engineering, vol. 14, no. 1, pp. 299-313, 2017.

[12] J. Fu, R. Ma, and T. Chai, "Global finite-time stabilization of a class of switched nonlinear systems with the powers of positive odd rational numbers," Automatica, vol. 54, pp. 360-373, 2015.

[13] X. Zhao, L. Zhang, P. Shi, and M. Liu, "Stability and stabilization of switched linear systems with mode-dependent average dwell time," IEEE Transactions on Automatic Control, vol. 57, no. 7, pp. 1809-1815, 2012.

[14] L. Xu, Q. Wang, W. Li, and Y. Hou, "Stability analysis and stabilisation of full-envelope networked flight control systems: switched system approach," IET Control Theory and Applications, vol. 6, no. 2, pp. 286-296, 2012.

[15] W. Ren and J. L. Xiong, "Stability and stabilization of switched stochastic systems under asynchronous switching," Systems \& Control Letters, vol. 97, pp. 184-192, 2016.

[16] H. B. Chen, P. Shi, and C. C. Lim, "Stability of neutral stochastic switched time delay systems: an average dwell time approach," International Journal of Robust and Nonlinear Control, vol. 27, no. 3, pp. 512-532, 2017.

[17] B. Niu, H. R. Karimi, H. Q. Wang, and Y. L. Liu, "Adaptive output-feedback controller design for switched nonlinear stochastic systems with a modified average dwell-time method," IEEE Transactions on Systems, Man, and Cybernetics: Systems, 2016.

[18] X. Wang and J. Zhao, "Switched adaptive tracking control of robot manipulators with friction and changing loads," International Journal of Systems Science, vol. 46, no. 6, pp. 955-965, 2015.

[19] L. Tang and J. Zhao, "Neural network based adaptive prescribed performance control for a class of switched nonlinear systems," Neurocomputing, vol. 230, pp. 316-321, 2017.

[20] B. Jiang, Q. Shen, and P. Shi, "Neural-networked adaptive tracking control for switched nonlinear pure-feedback systems under arbitrary switching," Automatica, vol. 61, pp. 119-125, 2015.

[21] L. Vu, D. Chatterjee, and D. Liberzon, "Input-to-state stability of switched systems and switching adaptive control," Automatica, vol. 43, no. 4, pp. 639-646, 2007.

[22] T.-T. Han, S. S. Ge, and T. H. Lee, "Adaptive neural control for a class of switched nonlinear systems," Systems \& Control Letters, vol. 58, no. 2, pp. 109-118, 2009.

[23] C. Hu, B. Yao, and Q. Wang, "Performance-oriented adaptive robust control of a class of nonlinear systems preceded by unknown dead zone with comparative experimental results,"
IEEE/ASME Transactions on Mechatronics, vol. 18, no. 1, pp. 178$189,2013$.

[24] J. Na, "Adaptive prescribed performance control of nonlinear systems with unknown dead zone," International Journal of Adaptive Control and Signal Processing, vol. 27, no. 5, pp. 426446, 2013.

[25] F. Yu, Z. Mao, and M. Jia, "Recursive identification for Hammerstein-Wiener systems with dead-zone input nonlinearity," Journal of Process Control, vol. 23, no. 8, pp. 1108-1115, 2013.

[26] S. Ibrir, W. F. Xie, and C.-Y. Su, "Adaptive tracking of nonlinear systems with non-symmetric dead-zone input," Automatica, vol. 43, no. 3, pp. 522-530, 2007.

[27] J. Zhao and D. J. Hill, "On stability, L2-gain and Ho control for switched systems," Automatica, vol. 44, no. 5, pp. 1220-1232, 2008.

[28] H. Y. Cho and E. R.-W. Bai, "Convergence results for an adaptive dead zone inverse," International Journal of Adaptive Control and Signal Processing, vol. 12, no. 5, pp. 451-466, 1998.

[29] X. Zhao, H. Yang, H. R. Karimi, and Y. Zhu, "Adaptive neural control of MIMO nonstrict-feedback nonlinear systems with time delay," IEEE Transactions on Cybernetics, vol. 46, no. 6, pp. 1337-1349, 2016.

[30] S. Yin, P. Shi, and H. Yang, "Adaptive fuzzy control of strict-feedback nonlinear time-delay systems with unmodeled dynamics," IEEE Transactions on Cybernetics, vol. 46, no. 8, pp. 1926-1938, 2016.

[31] Z. Liu, B. Chen, and C. Lin, "Adaptive neural backstepping for a class of switched nonlinear system without strict-feedback form," IEEE Transactions on Systems, Man, and Cybernetics: Systems, 2016.

[32] Q. Yang and S. Jagannathan, "Reinforcement learning controller design for affine nonlinear discrete-time systems using online approximators," IEEE Transactions on Systems, Man, and Cybernetics, Part B: Cybernetics, vol. 42, no. 2, pp. 377-390, 2012. 


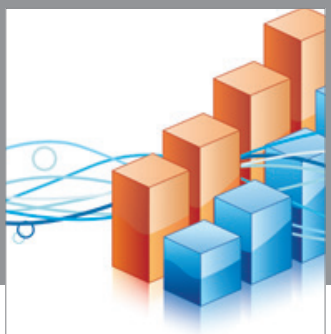

Advances in

Operations Research

vatem alat4

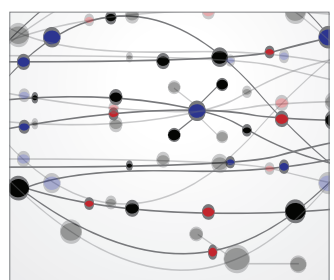

\section{The Scientific} World Journal
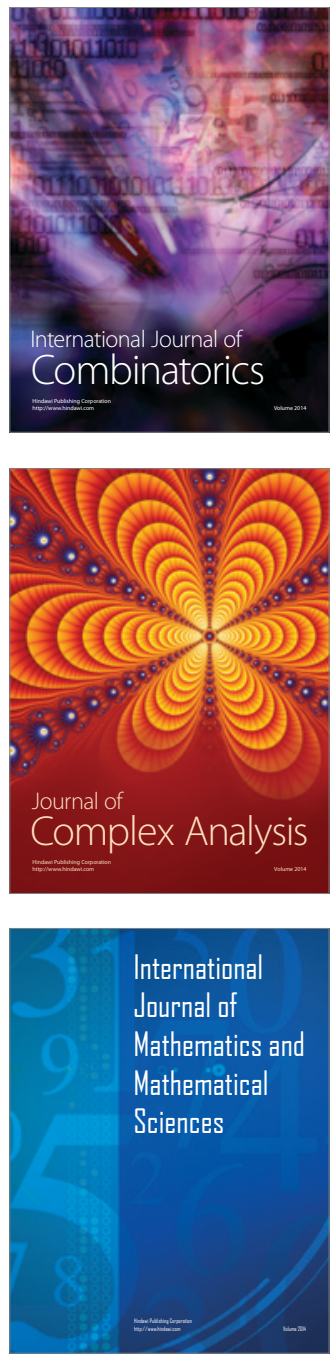
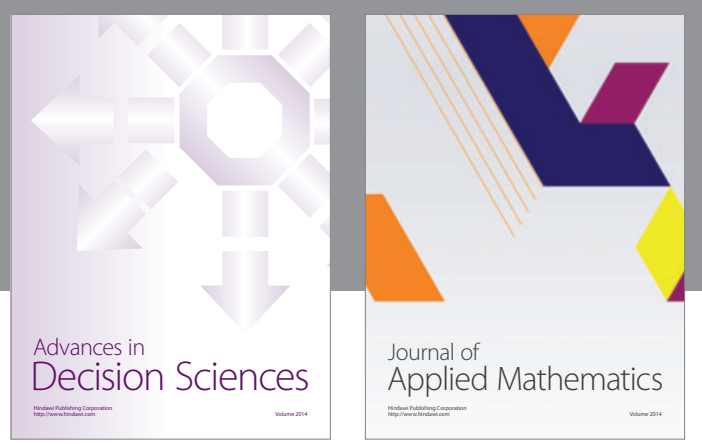

Algebra

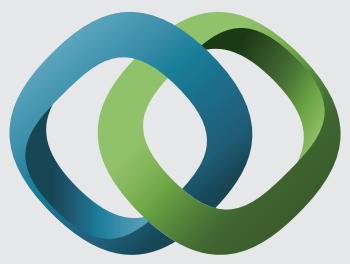

\section{Hindawi}

Submit your manuscripts at

https://www.hindawi.com
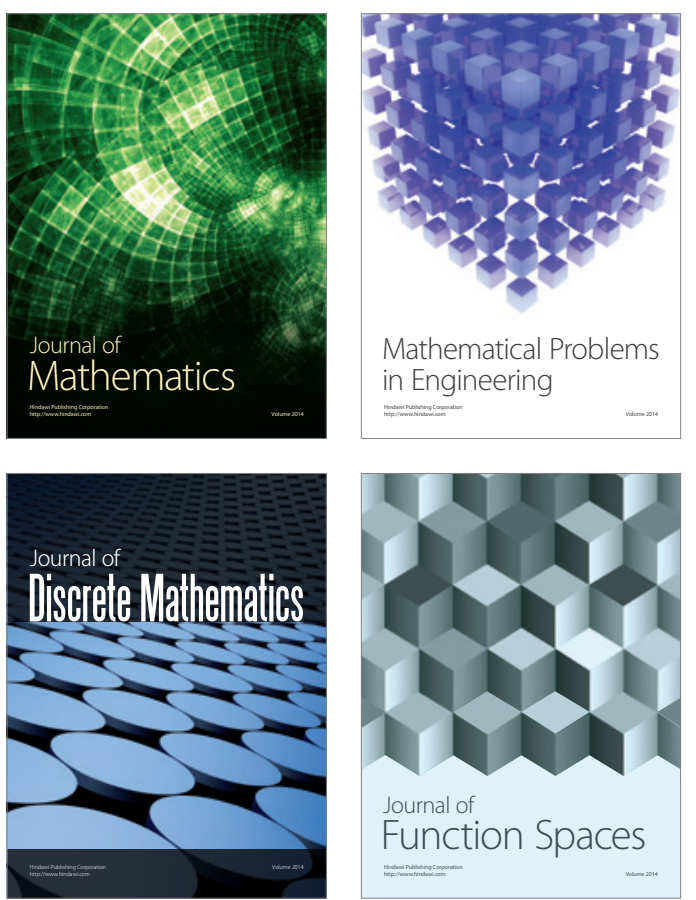

Mathematical Problems in Engineering
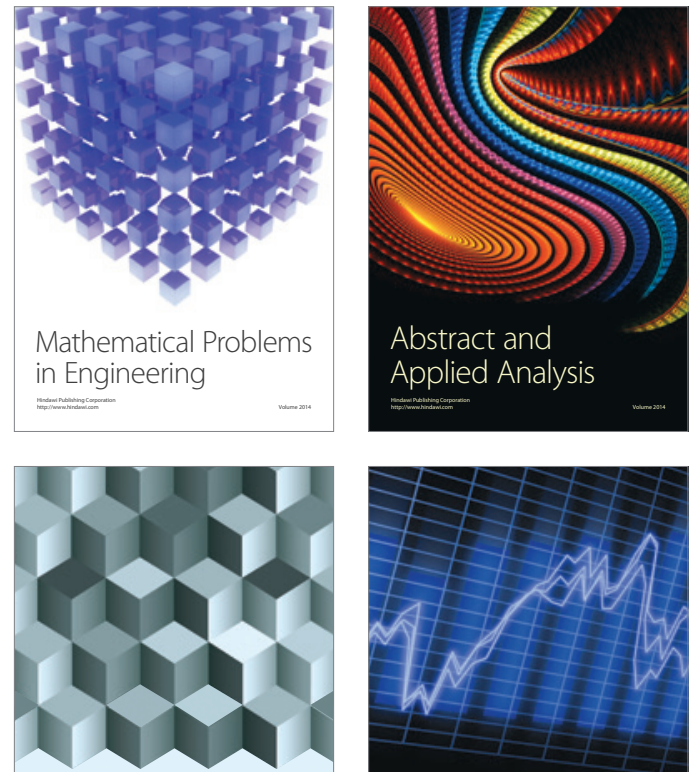

Journal of

Function Spaces

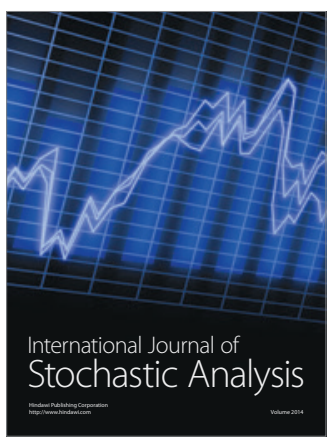

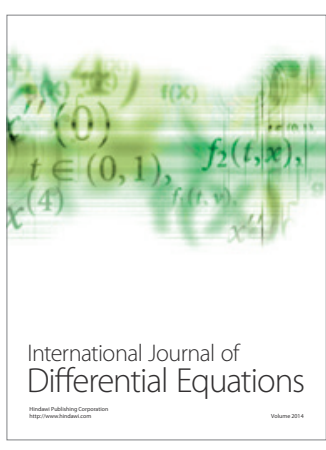
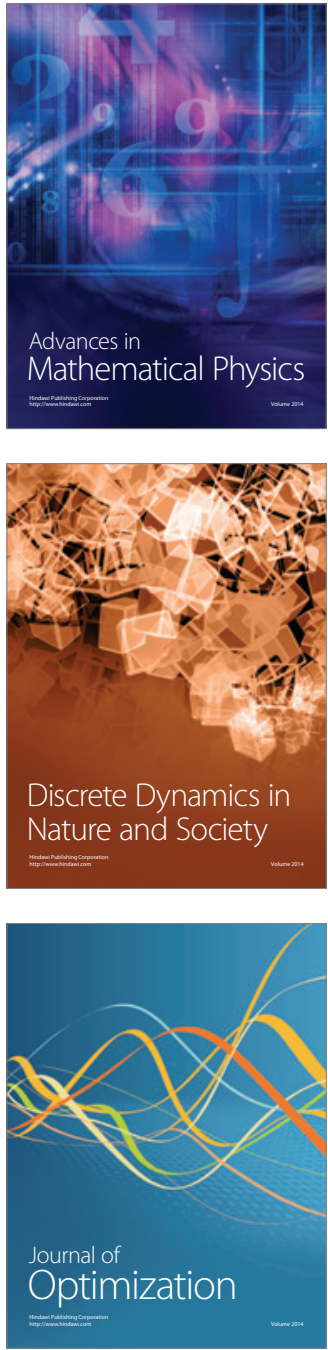